\title{
Factors influencing portal vein velocity after liver transplantation
}

Popescu M. ${ }^{1,2}$, Dima S. ${ }^{2}$, Marcu A. ${ }^{3}$, Calancea E. ${ }^{3}$, Dinca A. ${ }^{3}$, Tomescu D. ${ }^{1,2}$

'Carol Davila University of Medicine and Pharmacy, Dept of Anaesthesiology \& Intensive Care, Bucharest, Romania,

${ }^{2}$ Fundeni Clinical Institute, Dept of Surgery, Bucharest, Romania,

${ }^{3}$ Fundeni Clinical Institute, Dept of Anaesthesiology \& Intensive Care, Bucharest, Romania

\section{Introduction}

Vascular complications are one of the most common complications after liver transplantation (LT) and are generally associated with a poor prognosis if adequate measures are not undertaken. The aim of our study was to determine risk factors for decreased portal vein velocity and portal vein thrombosis after LT.

\section{Methods}

We retrospectively analyzed 165 patients undergoing deceased-donor LT. Preoperative severity (MELD and MELDsodium score) and thrombotic complications of liver disease, intraoperative blood loss and transfusion requirements were noted. Standard coagulation tests (INR, APTT, PT), platelet count and fibrinogen levels were recorded 15 minutes after reperfusion of the graft and for the first 3 postoperative days. Rotational thromboelastometry (ExTEM, InTEM, FibTEM, ApTEM) was performed 15 minutes into the neohepatic phase and 24 hours after LT and both standard parameters (clotting time-CT, clot formation time-CFT, maximum clot firmness-MCF) and derived (thrombin potential index-TPI, maximum velocity of clot formation-MaxV, time to MaxV-MaxVt, area under the curve-AUC and maximum clot elasticity-MCE) were noted. Maximum blood velocity in the portal vein (PVV) was determined daily during the first 3 postoperative days using Doppler ultrasound and the mean value was recorded. Portal vein thrombosis (PVT) was diagnosed using contrast-enhanced computer tomography.

\section{Results}

The mean age in the study group was $50 \pm 12$ years and the mean severity scores (MELD, MELD-sodium) were 19.2 \pm 6.1 and $21.5 \pm 6.0$ respectively. 23 patients (I4.2\%) were preoperatively diagnosed with PVT. Mean postoperative PVV was $44.9 \pm 15.9 \mathrm{~cm} / \mathrm{s}$ and 9 patients (5.5\%) were diagnosed with PVT. Risk factors for decreased postoperative PVV were high blood loss $(p=0.01)$, fresh frozen plasma transfusion $(p=0.02)$, intraoperative ExTEM CT $(p=0.01), \operatorname{InTEM~CT}$ $(p=0.01)$, ExTEM CFT $(p=0.01)$, ExTEM MCF $(p=0.02)$, AUC $(p=0.01)$ and postoperative ExTEM CT $(p=0.01), \operatorname{InTEM~CT}$ $(p=0.01)$.

Risk factors for postoperative PVT were: fresh frozen plasma transfusion $(p=0.0 \mathrm{I})$ and platelet transfusion $(p=0.0 \mathrm{I})$, intraoperative ExTEM CFT $(p=0.01)$, ExTEM MCF $(p=0.03)$ and postoperative ExTEM CT $(p=0.01)$, MaxVt $(p=0.01)$, AUC $(p=0.03)$.
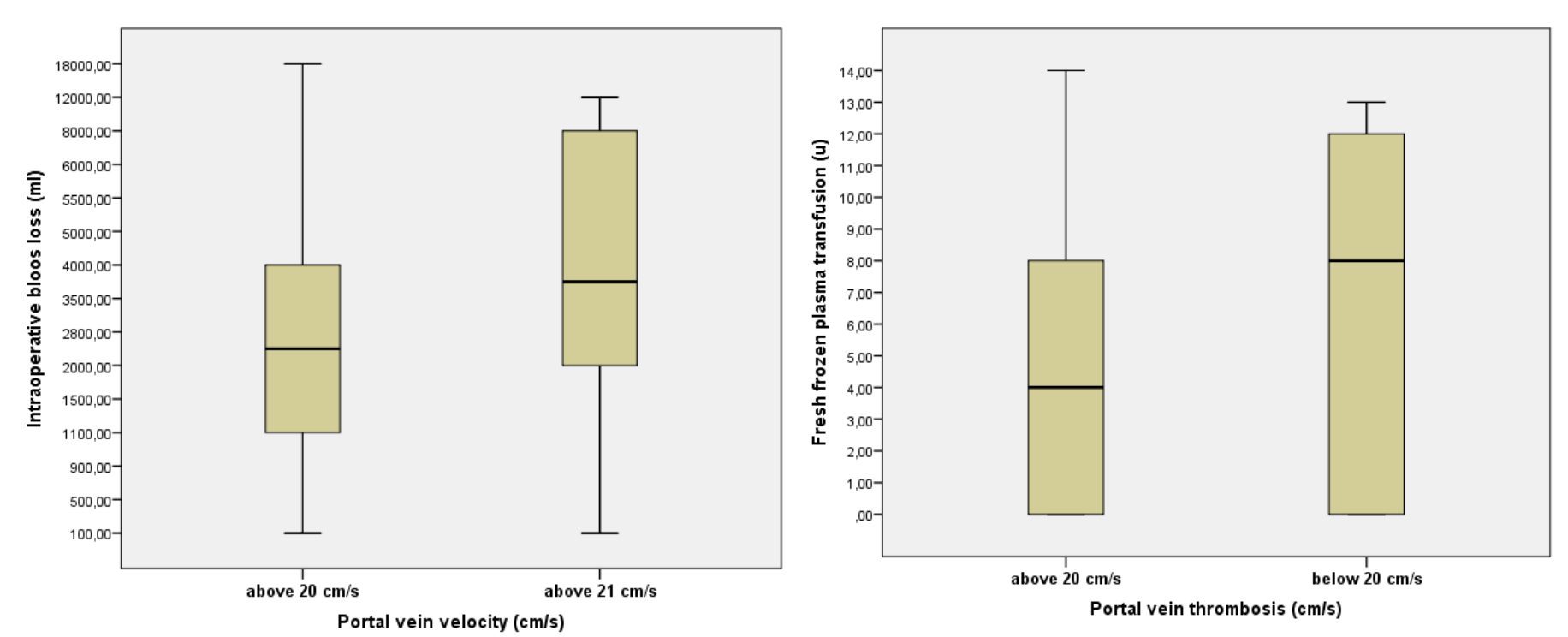

\section{Conclusion}

Blood loss and transfusion represent the most important and potentially reversible risk factors for both PVT and low PVV. Thromboelastometric parameters, especially extrinsically activated, may help in identifying patients at risk for PVT. 